\title{
Existence results for a new class of generalized implicit fuzzy resolvent dynamical systems in Banach spaces
}

\author{
Vo Minh Tam¹, Nguyen Van Hung ${ }^{2}$, and D. Baleanu ${ }^{3}$ \\ ${ }^{1}$ Dong Thap University \\ ${ }^{2}$ Ton Duc Thang University \\ ${ }^{3}$ Cankaya University
}

May 5, 2020

\begin{abstract}
In this paper, we investigate a new class of generalized implicit fuzzy resolvent dynamical systems involving $\$(\backslash$ mathbf $\{\mathrm{H}\}(\backslash$ cdot, $\backslash$ cdot $), \backslash$ eta $) \$-$ monotone operators in Banach spaces. Employing the approach of Gronwall's inequality, generalized resolvent mapping technique and neural network technique, we prove the existence of solutions for this type of systems under some suitable conditions. The results presented in this paper generalize and improve some corresponding results in the literature.
\end{abstract}

\section{Hosted file}

Existence_solutions-for-RDS-to-MMAS.pdf available at https://authorea.com/users/300160/ articles/429854-existence-results-for-a-new-class-of-generalized-implicit-fuzzyresolvent-dynamical-systems-in-banach-spaces 


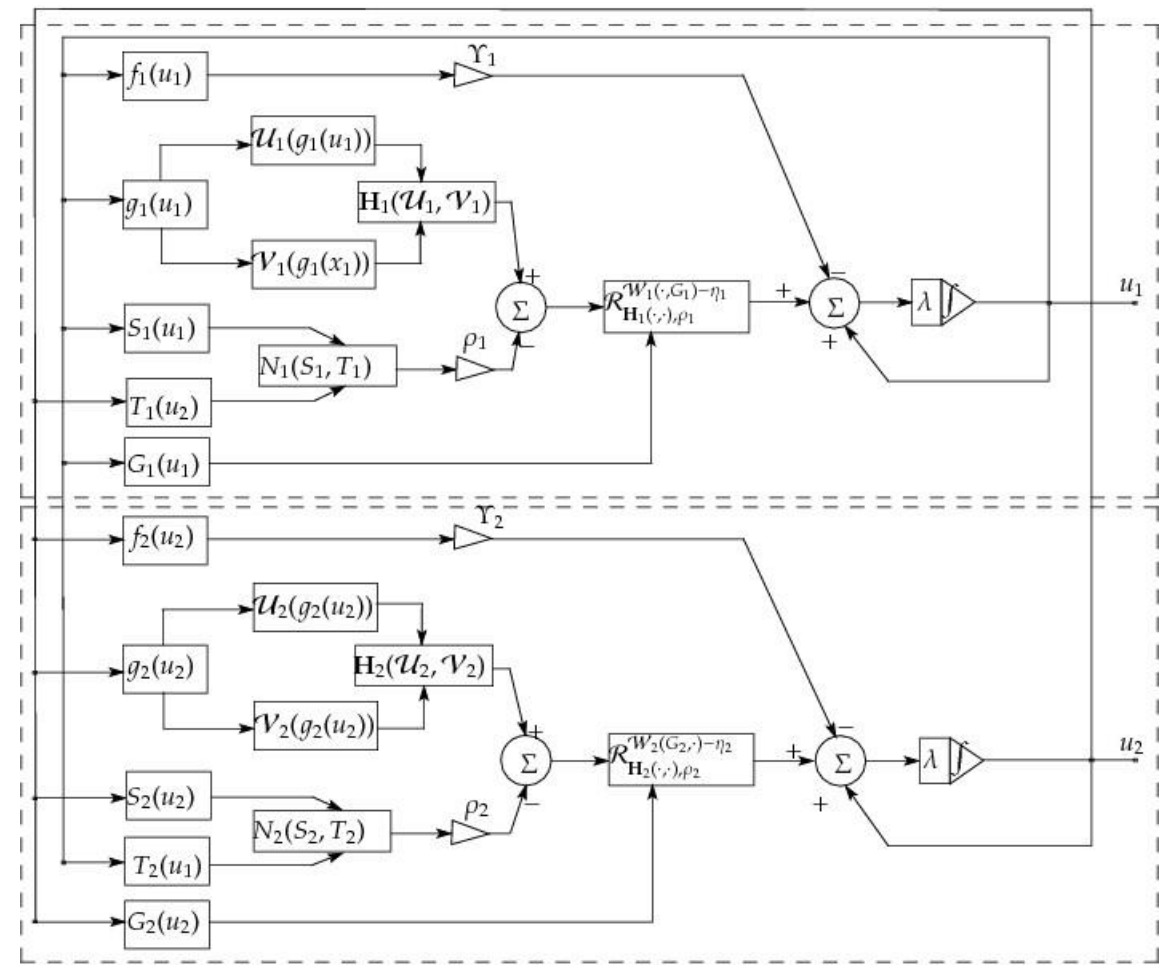

